Mazur Karol, Machaj Dominik, Mazur Dominika, Baran Marlena, Placzek Alicja. The use of peppermint oil in the treatment of irritable bowel syndrome. Journal of Education, Health and Sport. 2020;10(6):28-32. eISSN 2391-8306. DOI http://dx.doi.org/10.12775/JEHS.2020.10.06.003

https://apcz.umk.pl/czasopisma/index.php/JEHS/article/view/JEHS.2020.10.06.003

https://zenodo.org/record/3879955

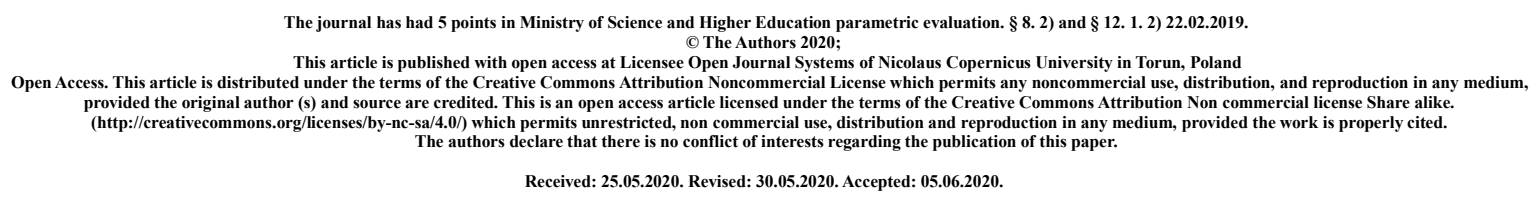

\title{
The use of peppermint oil in the treatment of irritable bowel syndrome
}

\author{
Karol Mazur, Dominik Machaj, Dominika Mazur, Marlena Baran, Alicja Placzek
}

Karol Mazur, mazurkarol79@gmail.com, Faculty of Medicine, Medical University of Lublin, Chodźki Street 19, 20-093 Lublin, Poland

Dominik Machaj, dominik5a4@tlen.pl, Faculty of Medicine, Medical University of Lublin, Chodźki Street 19, 20-093 Lublin, Poland

Dominika Mazur, dominika.hul20@gmail.com, Medical Faculty, University of Rzeszow, Pigonia Street 6, 35-310 Rzeszow, Poland

Marlena Baran, mbaran96@gmail.com, Medical Faculty, University of Rzeszow, Pigonia Street 6, 35-310 Rzeszow, Poland

Alicja Płaczek, alicja60@poczta.onet.pl, Medical Faculty, University of Rzeszow, Pigonia Street 6, 35-310 Rzeszow, Poland

\section{Summary:}

Irritable bowel syndrome (IBS) is a a chronic functional gastrointestinal disorder, that affects large intestine. The symptoms of IBS include: abdominal pain, discomfort, change in bowel habits and disordered defecation. The causes of irritable bowel syndrome have not yet been fully investigated and determined.

Peppermint (Mentha ×piperita) is a hybrid type of mint, a cross between watermint (Mentha aquatica) and spearmint (Mentha spicata). Peppermint oil is produced in the proces of steam distillation of fresh leaves of peppermint.

The aim of this study was to evaluate the use of peppermint oil in the treatment of irritable bowel syndrome. Our study material consisted of publications, which were found in PubMed, ResearchGate and Google Scholar databases. The first step was to find proper publications from the last 30 years. The second step was to carry out an overview of the found publications.

Based on the results of mentioned studies it can be concluded that peppermint oil is effective in the treatment of irritable bowel syndrome due to providing global symptom improvement and significant reduction of abdominal pain, and its short-term use is safe. However, further research is needed to estabilish the role of peppermint oil in the treatment of irritable bowel syndrome and to determine the optimal duration of treatment, dosage of peppermint oil, and its possible side effects related to long-term usage.

Key words: irritable bowel syndrome, IBS, peppermint, peppermint oil. 


\section{INTRODUCTION AND PURPOSE}

Irritable bowel syndrome (IBS) is a a chronic functional gastrointestinal disorder, that affects large intestine. The most common symptoms of IBS are: abdominal pain, discomfort, change in bowel habits and disordered defecation. Abdominal pain or discomfort associated is often poorly localized, migratory, and variable. Other symptoms of IBS include: bloating, abdominal distension, mucous in the stool, urgency, nausea, loss of appetite, and a feeling of incomplete evacuation. All of these symptoms may occur after a meal, during stress or at the time of menses [1]. The diagnosis of irritable bowel syndrome is made on the basis of recognition clinical symptoms and exclusion of other diseases. It is necessary to perform various medical diagnostic tests such as blood tests, stool tests, lactose intolerance test, hydrogen breath test, sigmoidoscopy, colonoscopy, and barium enema to exclude other organic diseases, which may give similar symptoms. The differential diagnosis should include: colon cancer, Crohn's disease, ulcerative colitis, hyperthyroidism, hypothyroidism, and giardiasis. The causes of irritable bowel syndrome have not yet been fully investigated and determined. However, it is believed that IBS can occur as a result of action of various factors, including visceral hypersensitivity, altered bowel motility, neurotransmitters imbalance, infection and psychosocial factors [2].

The aim of this study was to evaluate the use of peppermint oil in the treatment of irritable bowel syndrome. Our study material consisted of publications, which were found in PubMed, ResearchGate and Google Scholar databases. In order to find the proper publications, the search has been conducted with the use of a combination of key words like: "irritable bowel syndrome, "IBS", "peppermint", "peppermint oil". The first step was to find proper publications from the last 40 years. The second step was to carry out an overview of the found publications.

\section{DESCRIPTION OF THE STATE OF KNOWLEDGE}

Peppermint (Mentha ×piperita) is a hybrid type of mint, a cross between watermint (Mentha aquatica) and spearmint (Mentha spicata). Peppermint oil is produced in the process of steam distillation of fresh leaves of peppermint. It is available in the forms of essential oils, extracts, and capsules. The main constituents of peppermint oil are: menthol (40.7\%) menthone (23.4\%), menthyl acetate, 1,8-cineole, limonene, beta-pinene and betacaryophyllene [3]. Peppermint oil is used in the treatment of gastrointestinal disorders such as irritable bowel syndrome, nausea, flatulence, diarrhea and other conditions like headache, common cold, itchiness, allergic rash. It is also used in the production of food as a flavoring agent, dietary supplements, cosmetics such as soaps, shampoos, conditioners, bath salts and dental products, especially mouthwashes and toothpastes [4]. There are many studies that proved the efficacy of the peppermint oil in the treatment of irritable bowel syndrome.

Cash et al. in their trial investigated the role of the peppermint in the alleviating symptoms of irritable bowel syndrome. 72 patients, that were diagnosed with IBS according to the Rome III criteria, received orally either one capsule of IBgard ${ }^{\circledR}$ (containing $180 \mathrm{mg}$ of peppermint oil) or placebo three times a day before meal for 4 weeks. Those who received peppermint oil experienced statistically significant global improvement in symptoms of irritable bowel syndrome and reduction of abdominal pain. Authors also reported the 
occurrence of mild and transient treatment-related adverse reactions such as heartburn, flatulence, or dyspepsia in patients [5].

Merat et al. in their study evaluated the role of peppermint oil in the treatment of irritable bowel syndrome. 90 patients, that were diagnosed with IBS according to the Rome II criteria, received either one capsule of Colpermin (containing $187 \mathrm{mg}$ of peppermint oil) or placebo three times a day before meal for 8 weeks. Those who received peppermint oil experienced statistically significant reduction of abdominal pain. Authors also reported the occurrence of mild and transient treatment-related adverse reactions such as heartburn or skin rash in patients [6].

Liu et al. in their trial checked the efficacy of peppermint oil in the treatment of irritable bowel syndrome. 110 patients, that were diagnosed with IBS according to clinical diagnosis, received orally either one capsule of Colpermin (containing $187 \mathrm{mg}$ of peppermint oil) or placebo three to four times a day before meal for 4 weeks. Those who received peppermint oil experienced statistically significant global improvement in symptoms of irritable bowel syndrome and reduction of abdominal pain. Authors also reported the occurrence of mild and transient treatment-related adverse reactions such as heartburn or skin rash in patients [7].

Capello et al. in their study also assessed the role of peppermint oil in the alleviating symptoms of irritable bowel syndrome. 57 patients, that were diagnosed with IBS according to the Rome II criteria, received orally either two capsules of Mint oil (each containing 225 mg of peppermint oil) or placebo two times a day before meal for 4 weeks Those who received peppermint oil experienced statistically significant global improvement in symptoms of irritable bowel syndrome and reduction of abdominal pain. Authors also reported the occurrence of mild and transient treatment-related adverse reactions such as heartburn, headache, or dizziness in patients [8].

Capanni et al. in their study investigated the efficacy of peppermint oil in the treatment of irritable bowel syndrome. 178 patients that were diagnosed with IBS according to the Rome II criteria, received orally either two capsules of Mintoil (each containing $225 \mathrm{mg}$ of peppermint oil) or placebo three times a day before meal for 12 weeks. Those who received peppermint oil experienced statistically significant global improvement in symptoms of irritable bowel syndrome and reduction of abdominal pain. Authors also reported the occurrence of mild and transient treatment-related adverse reactions such as heartburn in patients [9].

Kline et al. in their trial checked the efficacy of peppermint oil in the treatment of irritable bowel syndrome in children. 42 patients, that were diagnosed with IBS according to Manning or Rome I criteria, received orally either one or two capsules of Colpermin (each containing $187 \mathrm{mg}$ of peppermint oil) or placebo three times a day before meal for 2 weeks. Those who received peppermint oil experienced statistically significant global improvement in symptoms of irritable bowel syndrome and reduction of abdominal pain. Authors observed no adverse reactions in patients [10].

Lech et al. in their trial evaluated the ability of peppermint oil to alleviate the symptoms of irritable bowel symptoms. 47 patients, that were diagnosed with IBS according to clinical diagnosis, received orally either $200 \mathrm{mg}$ of peppermint oil or placebo three times a day before meal for 4 weeks. Those who received peppermint oil experienced statistically 
significant global improvement in symptoms of irritable bowel syndrome and reduction of abdominal pain. Authors also reported the occurrence of mild and transient treatment-related adverse reactions such as heartburn in patients [11].

All the above mentioned studies proved that peppermint oil is effective in the treatment of irritable bowel syndrome. The mechanism of action of peppermint oil is thought to be causing the relaxation of gastrointestinal smooth muscle by blocking calcium channels, and thus decreasing gastrointestinal motility [12]. None of the above mentioned studies revealed any serious side effects. There was no statistically significant difference in reported adverse reactions between patients receiving peppermint oil and placebo. Furthermore reported side effects, the most common of which was heartburn, were mild and transient in nature. Based on the results of above mentioned studies it can be concluded that peppermint oil is effective in the treatment of irritable bowel syndrome due to providing global symptom improvement and significant reduction of abdominal pain, and its short-term use is safe. However, this conclusion is based on a small number of studies Additionally, there is large variation in the doses of oral peppermint oil and duration of treatment used in above mentioned studies. Because of that further research is needed to establish the role of peppermint oil in the treatment of irritable bowel syndrome and to determine the optimal duration of treatment, dosage of peppermint oil, and its possible side effects related to longterm usage [13].

\section{CONCLUSIONS}

1. All the above mentioned studies proved the effectiveness of peppermint oil in the treatment of irritable bowel syndrome.

2. None of the above mentioned studies revealed any serious side effects. Reported adverse reactions related to the usage of peppermint oil, the most common of which was heartburn, were mild and transient in nature.

3. Further research is needed to establish the role of peppermint oil in the treatment of irritable bowel syndrome and to determine the optimal duration of treatment, dosage of peppermint oil, and its possible side effects related to long-term usage.

\section{LIST OF REFERENCES:}

1. Alammar N, Wang L, Saberi B, et al. The impact of peppermint oil on the irritable bowel syndrome: a meta-analysis of the pooled clinical data. BMC Complement Altern Med. 2019;19(1):21.

2. Chey WD, Kurlander J, Eswaran S. Irritable bowel syndrome: a clinical review. JAMA. 2015;313(9):949-958.

3. Kligler B, Chaudhary S. Peppermint oil. Am Fam Physician. 2007;75(7):1027-1030.

4. Grigoleit HG, Grigoleit P. Gastrointestinal clinical pharmacology of peppermint oil. Phytomedicine. 2005;12(8):607-611.

5. Cash BD, Epstein MS, Shah SM. A novel delivery system of peppermint oil is an effective therapy for irritable bowel syndrome symptoms. Dig Dis Sci.2016;61(2):560-71.

6. Merat S, Khalili S, Mostajabi P, Ghorbani A, Ansari R, Malekzadeh R. The effect of enteric-coated, delayed-release peppermint oil on irritable bowel syndrome. Dig Dis Sci. 2010;55(5):1385-90. 
7. Liu JH, Chen GH, Yeh HZ, Huang CK, Poon SK. Enteric-coated peppermint-oil capsules in the treatment of irritable bowel syndrome: a prospective, randomized trial. $\mathrm{J}$ Gastroenterol. 1997;32(6):765-8.

8. Cappello G, Spezzaferro M, Grossi L, Manzoli L, Marzio L. Peppermint oil (Mintoil((R))) in the treatment of irritable bowel syndrome: a prospective double blind placebocontrolled randomized trial. Dig Liver Dis. 2007;39(6):530-6.

9. Capanni M, Surrenti E, Biagini MR, Milani S, Surrenh C, Galli A. Efficacy of peppermint oil in the treatment of irritable bowel syndrome: a randomized, controlled trial. Gazz med ital arch sci med [Internet]. 2005; 164(2):119-26.

10. Kline RM, Kline JJ, Di Palma J, Barbero GJ. Enteric-coated, pH-dependent peppermint oil capsules for the treatment of irritable bowel syndrome in children. J Pediatr. 2001;138(1):125-128.

11. Lech Y, Olesen KM, Hey H, Rask-Pedersen E, Vilien M, Ostergaard O. Treatment of irritable bowel syndrome with peppermint oil. A double-blind investigation with a placebo. Ugeskr Laeger. 1988;150(40):2388-9.

12. Hills JM, Aaronson PI. The mechanism of action of peppermint oil on gastrointestinal smooth muscle. Gastroenterology 1991;101:55-65.

13. Pietrzak A, Skrzydło-Radomańska B, Mulak A, et al. Guidelines on the management of irritable bowel syndrome: In memory of Professor Witold Bartnik. Prz Gastroenterol. 2018;13(4):259-288. 